\title{
DÜBLIN
}

Technological University Dublin

ARROW@TU Dublin

\section{Sensing of multiple parameters with whispering gallery mode optical fiber micro-resonators}

\author{
Arun Kumar Mallik Dr \\ arunkumar.mallik@tudublin.ie \\ Vishnan Kavungal \\ Technological University Dublin, vishnu.kavungal@tudublin.ie \\ Gerald Farrell \\ Technological University Dublin, gerald.farrell@tudublin.ie
}

See next page for additional authors

Follow this and additional works at: https://arrow.tudublin.ie/prccon

Part of the Electrical and Electronics Commons, Electromagnetics and Photonics Commons, and the Optics Commons

\section{Recommended Citation}

Arun Kumar Mallik, Vishnu Kavungal, Gerald Farrell, Yuliya Semenova, "Sensing of multiple parameters with whispering gallery mode optical fiber micro-resonators," Proc. SPIE 10904, Laser Resonators, Microresonators, and Beam Control XXI, 109041W (4 March 2019); https://doi.org/10.1117/12.2513412

This Conference Paper is brought to you for free and open access by the Photonics Research Centre at ARROW@TU Dublin. It has been accepted for inclusion in Conference Papers by an authorized administrator of ARROW@TU Dublin. For more information, please contact arrow.admin@tudublin.ie, aisling.coyne@tudublin.ie, gerard.connolly@tudublin.ie. Funder: Enterprise Ireland; European Regional Development Fund; Department of Agriculture, Food and the Marine

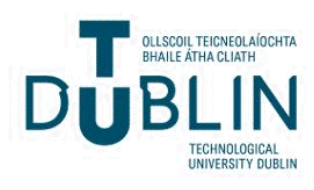




\section{Authors}

Arun Kumar Mallik Dr, Vishnan Kavungal, Gerald Farrell, and Yuliya Semenova

This conference paper is available at ARROW@TU Dublin: https://arrow.tudublin.ie/prccon/2 


\title{
Sensing of multiple parameters with whispering gallery mode optical fiber micro-resonators
}

\author{
Arun Kumar Mallik*, Vishnu Kavungal, Gerald Farrell and Yuliya Semenova* \\ Photonics Research Center, Technological University Dublin, Ireland
}

\begin{abstract}
Monitoring of multiple physical parameters, such as humidity, temperature, strain, concentrations of certain chemicals or gases in various environments is of great importance in many industrial applications both for minimizing adverse effects on human health as well as for maintaining production levels and quality of products. In this paper we demonstrate two different approaches to the design of multi-parametric sensors using coupled whispering gallery mode (WGM) optical fiber micro-resonators. In the first approach, a small array of micro-resonators is coupled to a single fiber taper, while in the second approach each of the micro-resonators within an array is coupled to a different tapered fiber section fabricated along the same fiber length. Simultaneous measurement of relative humidity and ammonia concentration in air is demonstrated with an array of two microspheres with different functional coatings coupled to a single fiber taper. Sensitivity to ammonia of $19.07 \mathrm{pm} / \mathrm{ppm}$ ammonia molecules and sensitivity to relative humidity of $1.07 \mathrm{pm} / \% \mathrm{RH}$ have been demonstrated experimentally. In the second approach, an inline cascade of two cylindrical micro-resonators fabricated by coupling to multiple tapered sections along a single optical fiber is demonstrated for measurement of strain and temperature simultaneously. A strain sensitivity of $1.4 \mathrm{pm} / \mu \varepsilon$ and temperature sensitivity of $330 \mathrm{pm} /{ }^{\circ} \mathrm{C}$ have been demonstrated experimentally. Both the proposed sensing systems have the potential for increase of the number of microresonators within an array for sensing of a larger number of parameters allowing for reduction of the overall cost of sensing system.
\end{abstract}

Keywords: Optical Resonators; Micro-optical Devices; Fiber Optic Sensors; Micro structured fibers

\section{INTRODUCTION}

Due to their high quality factors and low mode volumes whispering gallery mode (WGM) optical micro-resonators are capable of detecting minute changes in their size or surrounding refractive index with very high sensitivity. This has made them a well-established platform for highly sensitive physical, chemical and biological sensors of various parameters including refractive index (RI), temperature, force, electromagnetic fields and various chemicals and biological quantities. In the last 25 years WGM cavities of many different shapes, fabricated by a range of methods have been studied extensively, including fused silica microspheres [1-7], micro-droplets [8], micro-disks [9], micro-toroids [10], micro-rings [11], micro-cylinders [12], micro-bubbles [13], micro-spheroids [14], micro-pillbox [15], micro-gear [16], doughnutshaped [17], micro-coil [18], wheel shaped [19] and sausage-shaped structures [20]. Among these structures, microspheres and micro-cylindrical resonators are the simplest as they can be easily fabricated in laboratory conditions from a single piece of a standard silica fiber. The fabrication can be carried out without the need for sophisticated instruments or a cleanroom environment, which results in low fabrication costs, compared to other, more complex shapes made using mechanical polishing, microfiber manipulation or photolithographic patterning. Much of the research effort to date has been focused on using a single WGM resonator to sense a single physical, chemical or biological quantity, with only limited research focused on multi-parametric sensing [21]. However, in real life applications simultaneous sensing of multiple parameters is often required. Many effective techniques have been developed to realize multi-parametric measurements with fiber optic sensors. For example, simultaneous measurement of humidity and temperature has been proposed using a combination of a long period grating (LPG) inscribed in a fiber loop mirror [22], a combination of a Fiber Bragg Grating (FBG) and Fabry-Perot interferometer cavity [23], an FBG and photonics crystal fiber interferometer [24], an LPG partially coated with PAH/PAA poly electrolyte complexes [25], nafion-crystal violet film based optical sensor is to measurement of humidity and ammonia in air simultaneously [26], nano porous layer of alumina is also 
proposed for sensing of both humidity and ammonia gas [27] and many others. All the above mentioned sensing techniques rely on measurements of characteristic wavelength shifts in the spectra of the sensors influenced by the physical parameters of interest. Some of these configurations suffer from complexity of interrogation and a poor wavelength resolution. WGM micro resonators have the advantage over the above sensors due to their high Q-factors (up to $10^{9}$ ) [28], resulting in much higher wavelength resolution and low detection limits.

In this paper we propose and experimentally demonstrate two different approaches to the design of multi-parametric sensors using coupled WGM fiber micro-resonators for simultaneous sensing of multiple parameters. In the first approach we excite the WGMs in an array of two micro-spherical fiber resonators by coupling them to the same optical fiber taper. Two microspheres, $250 \mu \mathrm{m}$ and $255 \mu \mathrm{m}$ in diameter are used to measure ammonia concentration in air and humidity simultaneously. One of the microspheres was coated with a layer of silica sol-gel whose optical properties are very sensitive to the presence of ammonia, while the second microsphere was coated with a highly hygroscopic layer of $0.5 \%$ wt./vol. agarose hydrogel. An adiabatic tapered fiber with a uniform waist diameter of $\sim 4 \mu \mathrm{m}$ was used to simultaneously couple the light into both microspheres forming a small array. In the second approach an inline cascade of two cylindrical fiber micro-resonators (made from short sections of a polydimethylsiloxane (PDMS) and plastic optical fiber (POF)) was used to measure strain and temperature simultaneously. In contrast with the first approach, multiple tapered sections along the same optical fiber were used for excitation of WGMs in each of the micro-cylinders within the array. Here we discuss the fabrication, analyze the results of experimental investigations of both multi-parameter sensors and discuss the performance and advantages of each of the presented sensors.

\section{SENSOR ARRAYS FABRICATION AND EXPERIMENTAL SETUP}

\subsection{Fabrication of the WGM microsphere resonator array for relative humidity and ammonia sensing}

Two silica microsphere resonators were fabricated at the tips of single mode fibers using a standard fusion splicer in manual mode by applying a controlled number of electric arc discharges. The number of fusion splicer arcs was optimized to achieve a certain design diameter and a smooth surface of the microsphere sample. Fig. 1 shows microscopic images of a cleaved single mode fiber (left) and a fabricated $250 \mu \mathrm{m}$ diameter microsphere (right). Two microspheres with similar diameters $(250 \mu \mathrm{m}$ and $255 \mu \mathrm{m})$ were prepared for the experiments.

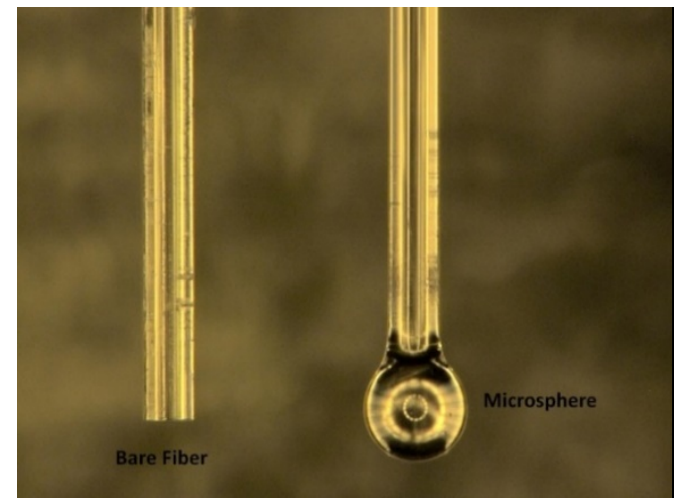

Figure 1. Optical microscope images of a bare fiber and a fabricated microsphere.

Two specific functional coatings were prepared and applied to the microspheres' surfaces in order to fabricate sensors of relative humidity $(\mathrm{RH})$ and ammonia concentration. Agarose hydrogel was utilized as the RH sensitive layer and a porous silica gel-coating was used to detect changes in the ammonia concentration in air. An agarose solution was firstly prepared by adding $0.05 \% \mathrm{wt}$./vol. of agarose powder into deionized (DI) water and the solution was constantly stirred at $80{ }^{\circ} \mathrm{C}$ until the agarose powder was completely dissolved in the DI water. Preparation of the sol-gel silica coating was carried out as described in [28]. The sol-gel was prepared by hydrolysis and condensation of TEOS (Tetraethyl ortho silicate) in the presence of water and ethanol. The molar ratios of TEOS, ethyl alcohol and DI water were 1:4:16 mixed in a 250-mL measuring flask and kept at continuous stirring conditions for 1.5 hour. 3-4 drops of hydrochloric acid were used as catalyst. The solution was kept for two days at room temperature. For each type of coatings, the influence of the coating thickness on the sensors' performance was investigated separately for both relative humidity and ammonia concentrations in the chamber at constant temperature. Dip coating method was used for application of coating layers, were both of the 
microspheres were pulled out of the respective solutions with the constant speed of $2 \mathrm{~mm} / \mathrm{sec}$. The thickness of the coatings was estimated as $300-400 \mathrm{~nm}$ for the silica gel and 500-600 $\mathrm{nm}$ for agarose by analyzing the corresponding SEM images of the samples.

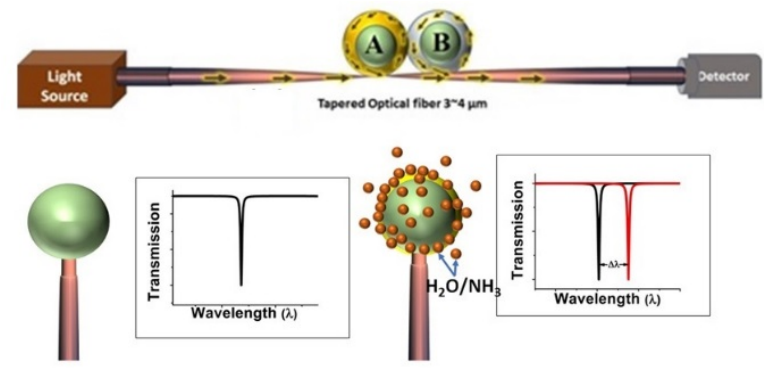

(a)

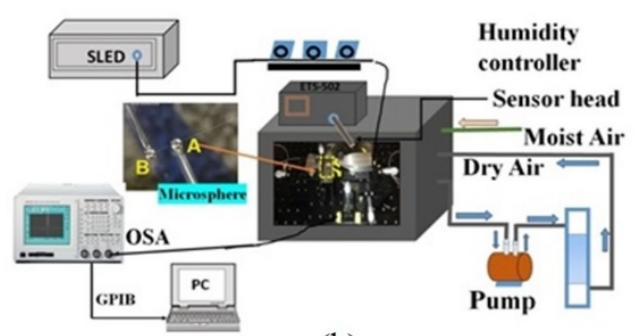

(b)

Figure 2. (a) Schematic diagram of the first approach (two microspheres are coupled to a single fiber taper); (b) Experimental setup for characterization of the $\mathrm{RH}$ and ammonia array sensor

Fig. 2 (a) schematically illustrates the two- microsphere sensor array and the basic detection principle. In the diagrams the silica gel coated microsphere is labelled as sensor A and the agarose coated microsphere as sensor B. Fig.2 (b) illustrates the experimental set up for characterization of the RH and ammonia array sensor consisting of a broadband light source, polarization controller and optical spectrum analyzer. All experiments were conducted in a humidity and temperature controlled chamber. To ensure efficient light coupling each of the microspheres were mounted on a XYZ micro-positioning stage, and then gradually and carefully brought in direct contact with the tapered fiber until the WGM resonances were clearly observed in the transmission spectrum of the fiber taper. In our experiment the tapered fiber waist diameter was approximately 3-4 microns. To improve mechanical stability, the fiber taper was fixed on a glass slide at a height of $5 \mathrm{~mm}$ from the slide surface using two drops of UV curable epoxy (Norrland).

\subsection{Inline cascaded array of WGM cylindrical micro resonators}

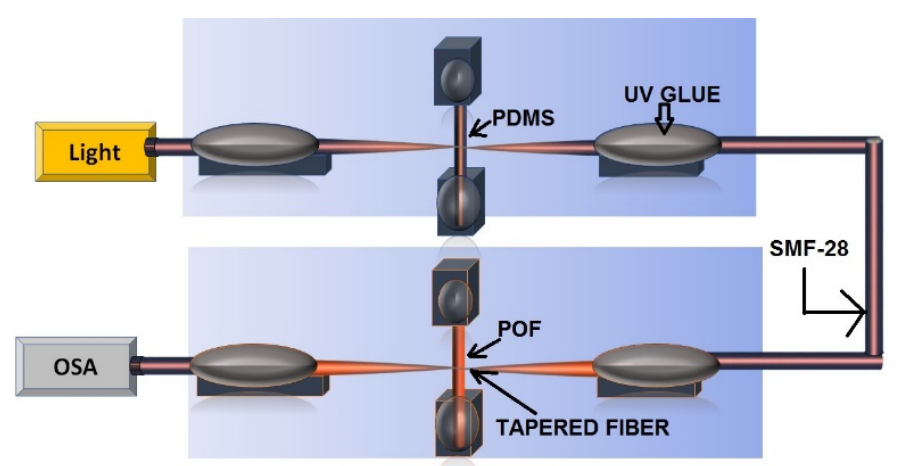

Figure 3. Schematic illustration of the cascaded inline two-micro-resonator array consisting of a PDMS-coated silica microcylinder and a POF micro-cylinder, coupled to different tapered sections along the same fiber.

Two micro-cylindrical resonators in our experiment were prepared from short sections of a standard coating-stripped silica fiber (SMF-28) and a polymer optical fiber (POF), whose diameters were reduced down to $10 \mu \mathrm{m}$ and $15 \mu \mathrm{m}$ respectively by heating and stretching. The silica fiber micro-cylinder was coated with a layer of polydimethylsiloxane (PDMS) and served as the temperature sensor. The PDMS material was chosen as the coating due to its low attenuation loss, good chemical stability and large thermo-optic coefficient [30]. The POF micro-cylinder served as the strain sensor because of the large elastic modulus of POF compared to silica fibers [31]. The PDMS coating was prepared as a solution with a base to curing agent ratio of 5:1 and the silica micro-cylinder was slowly pulled out of the prepared PDMS solution. After the application of the coating, the micro-resonator was cured at $80^{\circ} \mathrm{C}$ for 30 minutes and then at room temperature $\left(20-26^{\circ} \mathrm{C}\right)$ for further 24 hours. The thickness of the PDMS coating layer was estimated using optical microscopy to be circa 3.36 
$\mu \mathrm{m}$. The POF micro-resonator with a diameter of circa $15 \mu \mathrm{m}$ was prepared from a standard POF (GPOF 62, Thorlabs) with the diameter of $490 \mu \mathrm{m}[29]$.

The inline array of two cascaded cylindrical micro-resonators was constructed as shown schematically in Fig. 3. The PDMS and POF fiber cylinders were first fixed with a UV curable glue and then coupled to two separate tapered sections along the same fiber, whose ends were connected to the light source and an OSA.

\section{EXPERIMENTAL RESULTS AND DISCUSSION}

The experimental investigations of both sensor arrays were carried out with the help of a broadband light source (Thorlabs S5FC1005S), polarization controller, optical spectrum analyzer (OSA, Advantest Q8384) and a temperature controlled humidity chamber (ETS 5503). Light from the broadband light source operating in the wavelength range 1530-1570 nm was launched into the tapered fiber and the transmission spectra were observed at the fiber output by the OSA. The wavelength resolution of the OSA was $10 \mathrm{pm}$.

Excitation of WGMs in the micro-resonators was achieved by evanescent coupling to a tapered fiber. Fig. 4(a) illustrates transmission spectra of the taper for the RH-ammonia sensing array. At first, the agarose-coated microsphere (sensor B) was gradually and carefully brought in direct contact with the tapered fiber until the WGM resonances were clearly observed in the transmission spectrum of the fiber taper. The input light polarization was adjusted using a manual polarization controller to achieve maximum light coupling efficiency. Red curve (B) in Fig. 4 (a) illustrates the WGM spectrum of the tapered fiber when only sensor B is coupled. Without changing the setup, the second microsphere (sensor A) was positioned near the agarose-coated sphere and coupled to the same tapered fiber. The resulting transmission spectrum showing the WGM resonances due to both microspheres is labelled "A+B" in Fig. 4(a) (blue curve). The transmission spectrum of the taper when only sensor A is coupled is also shown in Fig. 4(a) (black curve), where the WGM resonances near $1540 \mathrm{~nm}$ can be clearly seen. As one can see from the figure, both groups of A and B resonances are clearly visible and distinguishable in the combined $\mathrm{A}+\mathrm{B}$ spectrum.

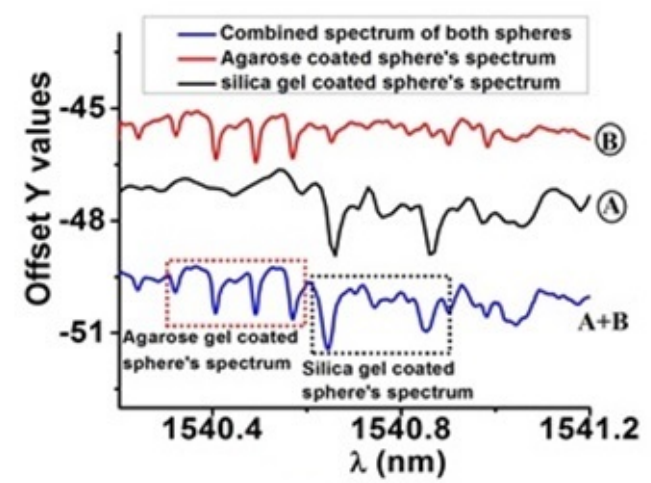

(a)

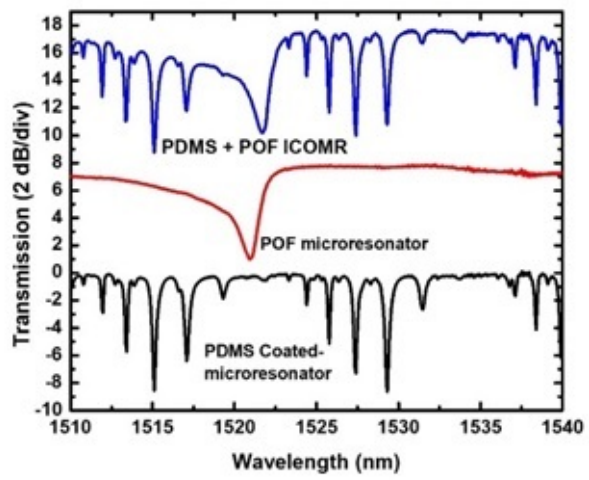

(b)

Figure 4. (a) WGM spectra of the silica gel and agarose coated sensors separately and when both microspheres are coupled simultaneously; (b) PDMS-coated micro-resonator, POF micro-resonator and the resulting combined spectrum.

Coupling of the two micro-cylindrical resonators within the strain-temperature cascaded array was carried out in a similar fashion. The corresponding WGM spectra excited by the individual resonators (PDMS-coated and POF) as well as the resulting combined spectrum are show in Fig. 4(b).

\subsection{Simultaneous measurement of RH and ammonia concentration using the two-microsphere-array}

The optical properties of the agarose coating are very sensitive to relative humidity, while those of the silica coating strongly depend on the ammonia concentration in the surrounding atmosphere and to a lesser extent on its relative humidity. Therefore changes in either of the parameters will result in spectral shifts of the WGM resonances associated with each of the coated microspheres (A and B), which can be expressed as: 


$$
\left[\begin{array}{l}
\Delta \lambda_{A} \\
\Delta \lambda_{B}
\end{array}\right]=\left[\begin{array}{ll}
K_{11} & K_{12} \\
K_{21} & K_{22}
\end{array}\right]\left[\begin{array}{c}
\Delta_{R H} \\
\Delta_{N H 3}
\end{array}\right]
$$

where $K_{11}$ and $K_{12}$ are the linear coefficients representing sensitivity of sensor A to relative humidity and ammonia concentration respectively. Similarly, $K_{21}$ and $K_{22}$ are the RH and ammonia sensitivities of sensor B.

Experimental measurements of humidity inside the experimental chamber were carried out for the sensor array in the range of relative humidity values from $40 \%$ to $70 \% \mathrm{RH}$ at a constant temperature of $25 \pm 0.1^{\circ} \mathrm{C}$. The sensing mechanism for both sensors $\mathrm{A}$ and $\mathrm{B}$ is based on the change of the effective refractive index and thickness of the coating layer due to adsorption of water by the coating surface for sensor A and ammonia vapor for sensor B. This process leads to the air inside the micro pores of the coating layer being replaced with water and ammonia molecules under capillary forces. As a result, the effective refractive index and thickness of the coating increases and WGMs experience a red spectral shift. The top graph in Fig. 5(a) represents the WGM spectra of the two-sensor array at different levels of relative humidity in the chamber. A commercial electronic hygrometer was used as a reference for humidity measurement inside the chamber. Bottom graph in Fig.5 (a) is a plot of the spectral shifts of the selected WGM resonances for sensors A and B as functions of relative humidity inside the chamber. One can see from the graph that both dependences are very close to linear and the corresponding sensitivities to humidity are $K_{11}=0.8 \mathrm{pm} / \% \mathrm{RH}$ for sensor A and $\mathrm{K}_{21}=1.07 \mathrm{pm} / \% \mathrm{RH}$ for sensor B. The same experimental setup was used to investigate the optical responses of the sensors A and B to different concentrations of ammonia vapors at constant temperature of $25 \pm 0.1{ }^{\circ} \mathrm{C}$ and constant humidity of $40 \% \mathrm{RH}$ inside the chamber. Small amounts of ammonium hydroxide $\left(\mathrm{NH}_{4} \mathrm{OH}\right)$ liquid were injected into and then removed from the chamber after the measurement, in order to create three low ammonia vapor concentrations corresponding to $0.46 \mathrm{ppm}, 1.46 \mathrm{ppm}$, and 2.19 ppm of ammonia in air. It should be noted that the WGM spectral shift dynamics with time depend on the diffusion rate of ammonia vapors inside the chamber.

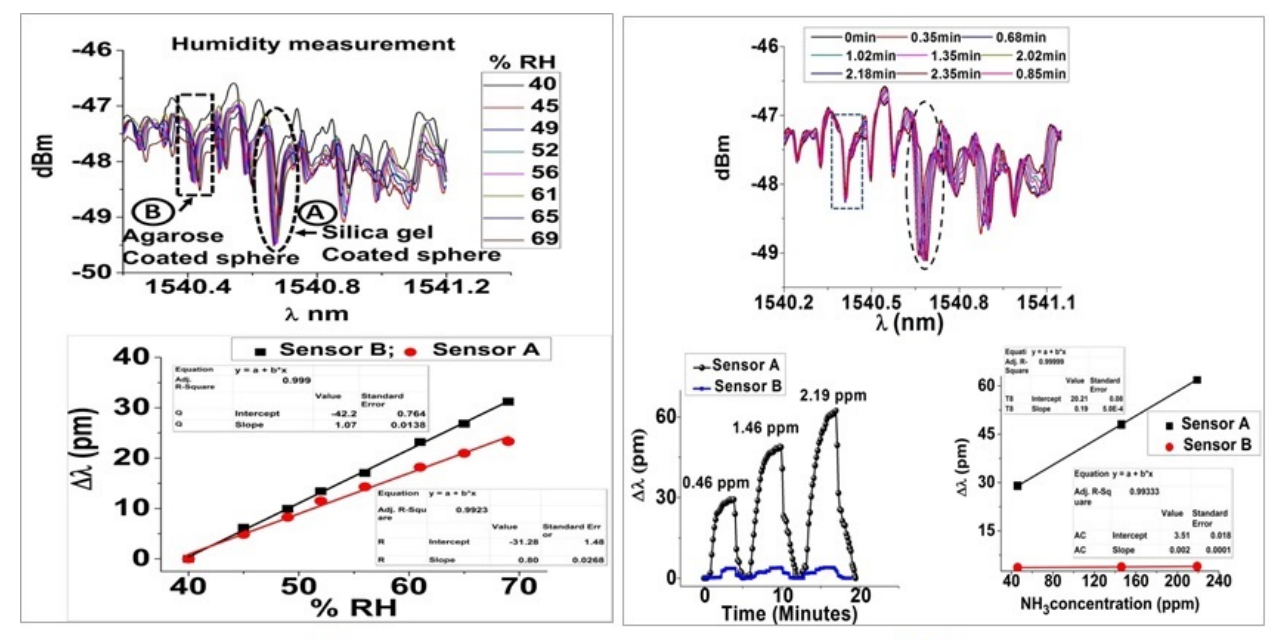

(a)

(b)

Top graph in Fig.5 (b) illustrates the spectra for the array, taken at different times after injection of ammonia vapor at 0.46 ppm concentration inside the chamber. As can be seen from the figure, the WGM resonance associated with the silica gel coated microsphere (dotted oval) experiences red shift during the sensor's exposure to ammonia vapor. The corresponding spectral shift of the WGM resonance associated with sensor B (dotted rectangle) also occurs in the direction towards the longer wavelengths but is much smaller. The bottom left graph in Fig.5 (b) illustrates the dynamics of the WGM spectral shifts for both the microspheres as they were exposed to $0.46 \mathrm{ppm}, 1.46 \mathrm{ppm}$ and $2.19 \mathrm{ppm}$ concentrations of ammonia inside the chamber. The bottom right graph in Fig.5 (b) shows that the total shift of the selected WGM resonance wavelength for sensor A was approximately 95 times larger than that for the sensor B during their exposure to $0.46 \mathrm{ppm}$ ammonia vapor concentration. The corresponding ammonia sensitivity coefficients $K_{12}$ and $K_{21}$ derived from the linear fits 
of the experimental data are $K_{12}=19.07 \mathrm{pm} / \mathrm{ppm}$ and $K_{22}=0.2 \mathrm{pm} / \mathrm{ppm}$ at constant temperature of $25 \pm 0.1^{\circ} \mathrm{C}$ and $40 \% \mathrm{RH}$ humidity.

By measuring the relevant WGMs spectral shifts, ammonia concentration in air and RH can be determined simultaneously. A study of the influence of temperature on the performance of the proposed sensor array was also carried out using the same environmental chamber and detailed experimental results were presented in our previous publication [33].

\subsection{Simultaneous measurement of strain and temperature using inline cascaded cylindrical resonators}

Experimental measurement of strain and temperature with the array of two cascaded cylindrical micro-resonators was carried out by placing the two sensors circa 1 meter apart from each other. The PDMS-coated resonator was mounted at the top of a temperature-controlled hot stage, and its temperature was gradually increased from room temperature $\left(25^{\circ} \mathrm{C}\right)$ to $45^{\circ} \mathrm{C}$ in $2{ }^{\circ} \mathrm{C}$ steps. The temperature of the hot stage was measured by a thermocouple with a $0.1^{\circ} \mathrm{C}$ measurement resolution. Strain was applied to the POF micro-resonator by axial elongation with a step of $0.01 \mathrm{~mm}$ using a translation stage with a resolution of $10 \mu \mathrm{m}$ as explained in [31]. To avoid the influence of temperature on the POF micro-resonator it was placed inside an environmental chamber maintaining constant room temperature. The resulting spectral changes were recorded using an OSA.

Figure 6 (a) shows the experimental transmission spectra of the array recorded at different temperatures and strains applied to the respective micro-resonators. In the figure, one of the groups of the WGM resonances associated with the PDMScoated silica micro-resonator is labelled 'PDMS-WGM' and the resonance due the POF micro-resonator is marked as 'POF-WGM'. It can be seen from the figure that an increase in temperature results in a blue shift of the PDMS-WGM

resonances and an increase in the applied axial strain also leads to a slight blue shift of the 'POF-WGM' spectral dip. In more detail, Fig. 6 (b) \& (c) illustrate the measured dependences of the spectral positions of selected WGM resonances associated with each of the micro-resonators as functions of the applied axial strain and temperature with the corresponding linear fittings. The PDMS-WGM resonances experienced a total spectral blue shift of $6.47 \mathrm{~nm}$ within the given temperature range resulting in estimated sensitivity of $330 \mathrm{pm} /{ }^{\circ} \mathrm{C}$. The POF-WGM experienced a total spectral shift of $0.99 \mathrm{~nm}$ within the studied tensile strain range from 0 to $714 \mu \varepsilon$ with a sensitivity of $1.4 \mathrm{pm} / \mu \varepsilon$ due to the combined effect of the decrease in diameter and the strain induced changes in the refractive index of the resonator as explained in [31].

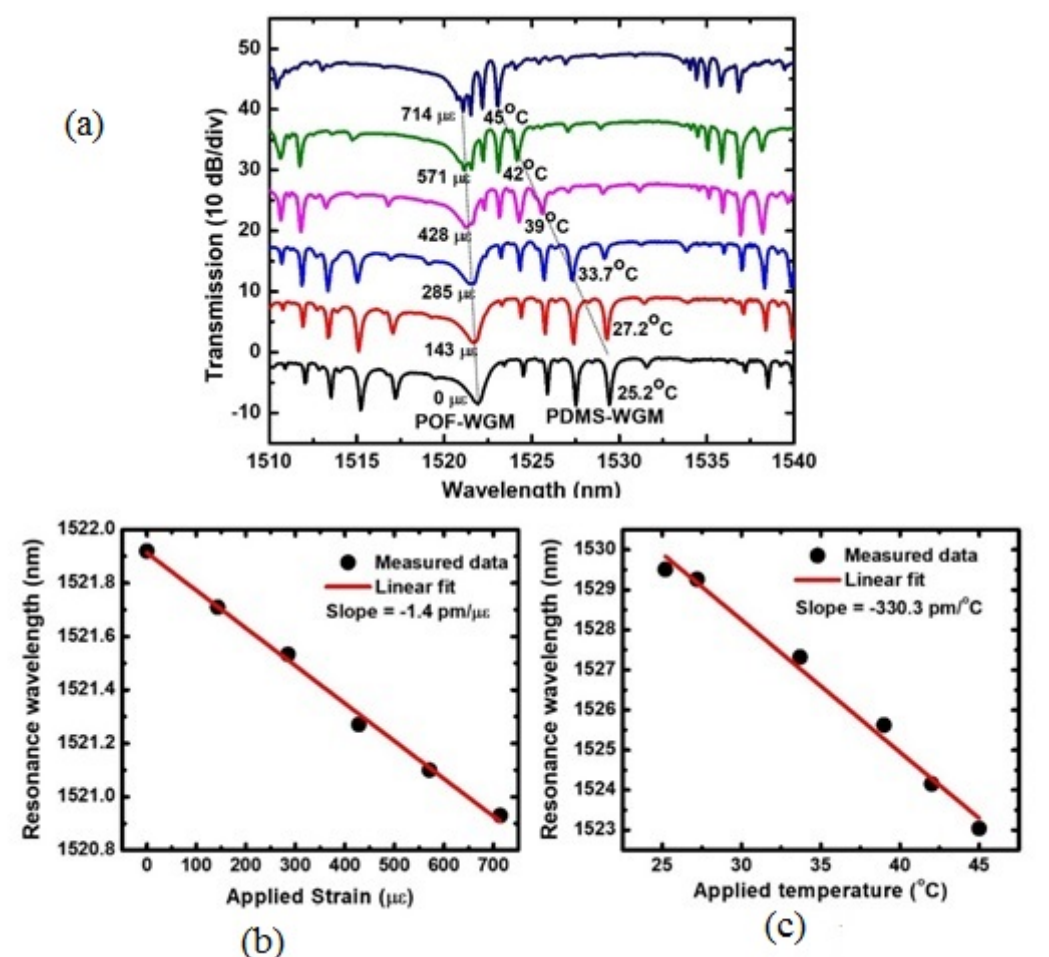

Figure 6. (a) WGM spectra for the array at different temperatures and strain values; (b) strain response of the POF microresonator; (c) temperature response of the PDMS-coated micro-resonator. 
resonances and an increase in the applied axial strain also leads to a slight blue shift of the 'POF-WGM' spectral dip. In more detail, Fig. 6 (b) \& (c) illustrate the measured dependences of the spectral positions of selected WGM resonances associated with each of the micro-resonators as functions of the applied axial strain and temperature with the corresponding linear fittings. The PDMS-WGM resonances experienced a total spectral blue shift of $6.47 \mathrm{~nm}$ within the given temperature range resulting in estimated sensitivity of $330 \mathrm{pm} /{ }^{\circ} \mathrm{C}$. The POF-WGM experienced a total spectral shift of $0.99 \mathrm{~nm}$ within the studied tensile strain range from 0 to $714 \mu \varepsilon$ with a sensitivity of $1.4 \mathrm{pm} / \mu \varepsilon$ due to the combined effect of the decrease in diameter and changes in the refractive index of the resonator as explained in [31].

\section{CONCLUSION}

In conclusion, two different approaches to the design of multi-parametric sensors using coupled WGM fiber microresonators have been proposed and demonstrated experimentally. In the first approach, a small array of micro-resonators is coupled to a single fiber taper, while in the second approach each of the micro-resonators within an array is coupled to a different tapered fiber section fabricated along the same fiber length. Simultaneous measurement of relative humidity and ammonia concentration in air demonstrated with an array of two microspheres with different functional coatings coupled to a single fiber taper. Sensitivity to ammonia of $19.07 \mathrm{pm} / \mathrm{ppm}$ and sensitivity to relative humidity of $1.07 \mathrm{pm} / \%$ RH have been demonstrated experimentally. In the second approach, an inline cascade of two cylindrical micro-resonators fabricated by coupling to multiple tapered sections along a single optical fiber has been demonstrated for simultaneous measurement of strain and temperature. Strain sensitivity of $1.4 \mathrm{pm} / \mu \varepsilon$ and temperature sensitivity of $330 \mathrm{pm} /{ }^{\circ} \mathrm{C}$ have been shown.

Both the proposed sensing systems have the potential for increase of the number of micro-resonators within an array for sensing of a larger number of parameters allowing for reduction of the overall cost of interrogation. It should be noted however that while the first approach is compact and can be used for point measurement of several parameters, the number of micro-resonators that can be used in array is limited due to the cross-sensitivity arising as a result of the physical proximity of multiple resonators. The second approach allows one to overcome the cross-sensitivity issue but is more suitable for a quasi-distributed sensing at different points in space.

\section{ACKNOLEDGEMENT}

Authors acknowledge the support of Enterprise Ireland and the European Regional Development Fund under Ireland's European Structural and Investment Funds Programme 2014-2020, Grant No CF-2016-0448-P and the Department of Agriculture, Food and the Marine's Competitive Research Funding Programmes, Grant No 17/F/284.

\section{REFERENCES}

[1]. N. M. Hanumegowda, C. J. Stica, B. C. Patel, I. White, and X. Fan, "Refractometric sensors based on microsphere resonators," Appl. Phys. Lett. 87, 1-3 (2005).

[2]. Q. Ma, T. Rossmann, and Z. Guo, "Whispering-gallery mode silica microsensors for cryogenic to room temperature measurement," Meas. Sci. Technol. 21 (2010).

[3]. T. Ioppolo, M. Kozhevnikov, V. Stepaniuk, M. V. Ötügen, and V. Sheverev, "Micro-optical force sensor concept based on whispering gallery mode resonators," Appl. Opt. 47, 3009-3014 (2008).

[4]. T. Ioppolo, U. Ayaz, and M. V. Otugen, "Tuning of whispering gallery modes of spherical resonators using an external electric field," Opt. Express 17, 16465-16479 (2009).

[5]. V. Ilchenko, P. Volikov, V. Velichansky, F. Treussart, V. Lefèvre-Seguin, J.-M. Raimond, and S. Haroche, "Straintunable high-Q optical microsphere resonator," Opt. Commun. 145, 86-90 (1998).

[6]. N. Lin, L. Jiang, S. Wang, Q. Chen, H. Xiao, Y. Lu, and H. Tsai, "Simulation and optimization of polymer-coated microsphere resonators in chemical vapor sensing," Appl. Opt. 50, 5465 (2011).

[7]. F. Vollmer, D. Braun, A. Libchaber, M. Khoshsima, I. Teraoka, and S. Arnold, "Protein detection by optical shift of a resonant microcavity," Appl. Phys. Lett. 80, 4057-4059 (2002)

[8]. M. Hossein-Zadeh and K. J. Vahala, "Fiber-taper coupling to WhisperingGallery modes of fluidic resonators embedded in a liquid medium," Opt. Express, 14, 10800-10810 (2006).

[9]. K. J .Vahala, "Optical microcavities," Nature 424, 839-846 (2003). 
[10]. M. Soltani, S. Yegnanarayanan, and A. Adibi, "Ultra-high Q planar silicon microdisk resonators for chip-scale silicon photonics," Opt. Express, 15, 4694- 4704 (2007).

[11]. $\quad$ K.Armani, T. J. Kippenberg, S. M. Spillane, and K. J. Vahala, "Ultra-high-Q toroid microcavity on a chip," Nature, 421, 925-928 (2003).

[12]. Q. Xu, D. Fattal, R. G. Beausoleil, "Silicon microring resonators with 1.5- $\mu$ m radius," Opt. Express, $16,4309-4315$ (2008).

[13]. X. Jin, Y. Dong, and K. Wang, "Selective excitation of axial modes in a highQ microcylindrical resonator for controlled and robust coupling," Appl. Opt. 54, 8100-8107 (2015).

[14]. J. M. Ward, Y. Yang, and S. N. Chormaic, "Highly Sensitive temperature measurements with liquidcore microbubble resonators," IEEE Photon. Technol. Lett. 25, 2350-2353 (2013).

[15]. M. Fujii, M. Haraguchi, T. Okamoto, and M. Fukui, "Characteristics of whispering gallery modes in single dielectric spheroid excited by Gaussian beam," Japanese J. Appl. Physics, 44, 4948-4955 (2005).

[16]. $\quad$ A. Ahmed, R. Koya, O. Wada, M. Wand, and R. Koga, "Field Analysis of pillbox resonator with radiation loss utilizing finite-element beam propagation method formulation," Opt. Rev. 3, 109-113 (1996).

[17]. $\quad$ A.B. Matsko, A. A. Savchenkov, and L. Maleki, "Vertically coupled whispering-gallery-mode resonator waveguide," Opt. Lett. 30, 3066-3068 (2005).

[18]. M. Sumetsky, "Whispering gallery modes in a microfiber coil with an n-fold helical symmetry: classical dynamics, stochasticity, long period gratings, and wave parametric resonance," Opt. Express, 18, 2413-25 (2010).

[19]. X. Sun, K. Y. Fong, C. Xiong, W. H. P. Pernice, and H. X. Tang, "GHz optomechanical resonators with high mechanical Q factor in air," Opt. Express, 19, 22316-22321 (2011).

[20]. Y. L. Shang, M. Y. Ye, and X.-M. Lin, "Experimental observation of Fano-like resonance in a whispering-gallery-mode microresonator in aqueous environment," Photonics Res. 5, 119-123 (2017).

[21]. F. Vollmer, S. Arnold, D. Braun, I. Teraoka, and A. Libchaber, "Multiplexed DNA quantification by spectroscopic shift of two microsphere cavities," Biophys. J. 85, 1974-1979 (2003).

[22]. H. Liu, H. Liang, M. Sun, K. Ni, and Y. Jin, "Simultaneous measurement of humidity and temperature based on a long-period fiber grating inscribed in fiber loop mirror," IEEE Sensors J. 14, 893-896 (2014).

[23]. F. J. Arregui, I. R. Matías, K. L. Cooper, and R. O. Claus, "Simultaneous measurement of humidity and temperature by combining a reflective intensity-based optical fiber sensor and a fiber Bragg grating," IEEE Sensors J. 2, 482-487 (2002).

[24]. J. Mathew, Y. Semenova, and G. Farrell, "Fiber optic hybrid device for simultaneous measurement of humidity and temperature," IEEE Sensors J. 13, 1632-1636 (2013).

[25]. A. Urrutia, J. Goicoechea, A. L. Ricchiuti, D. Barrera, S. Sales, and F. J. Arregui, "Simultaneous measurement of humidity and temperature based on a partially coated optical fiber long period grating," Sensors Actuators, B: Chem.227, 135-141 (2016).

[26]. I. M. Raimundo and R. Narayanaswamy, "Simultaneous determination of relative humidity and ammonia in air employing an optical fibre sensor and artificial neural network," in Sensors and Actuators, B: Chemical, (2001).

[27]. $\quad$ E. C. Dickey, O. K. Varghese, K. G. Ong, D. Gong, M. Paulose, and C. A. Grimes, "Room Temperature Ammonia and Humidity Sensing Using Highly Ordered Nanoporous Alumina Films," Sensors 2, 91-110 (2002).

[28]. M. L. Gorodetsky, A. A. Savchenkov, and V. S. Ilchenko, "Ultimate Q of optical microsphere resonators," Opt. Lett. 21, 453 (1996).

[29]. G. Brambilla, V. Finazzi, and D. J. Richardson, "Ultra-low-loss optical fiber nanotapers," Opt. Express, vol. 12, no. 10, p. 2258, 2004.

[30]. $\quad$ B. B. Li, Q. Y. Wang, Y. F. Xiao, X. F. Jiang, Y. Li, L. Xiao, and Q. Gong, "On chip, high-sensitivity thermal sensor based on high- Q polydimethylsiloxane-coated microresonator," Appl. Phys. Lett. 96, 2008- 2011 (2010).

[31]. V. Kavungal, G. Farrell, Q. Wu, A. K. Mallik, and Y. Semenova, "A packaged whispering gallery mode strain sensor based on a polymer wire cylindrical micro resonator," J. Light. Technol. 36, 1757-1765 (2017).

[32]. I. A. Grimaldi, S. Berneschi, G. Testa, F. Baldini, G. Nunzi Conti, and R. Bernini, "Polymer based planar coupling of self-assembled bottle microresonators," Appl. Phys. Lett. 105, 231114 (2014).

[33]. $\quad$ A. K. Mallik, G. Farrell, M. Ramakrishnan, V. Kavungal, D. Liu, Q. Wu, and Y. Semenova, "Whispering gallery mode micro resonators for multi-parameter sensing applications," Opt. Express 26, 31829-31838 (2018).

[34]. A. Grimaldi, S. Berneschi, G. Testa, F. Baldini, G. Nunzi Conti, and R. Bernini, "Polymer based planar coupling of self-assembled bottle microresonators," Appl. Phys. Lett. 105, 231114 (2014). 